\title{
Effects of soil settlement and deformed geometry on a historical structure
}

\author{
Y. Yardım and E. Mustafaraj \\ Department of Civil Engineering, Epoka University, Tirana, Albania \\ Correspondence to: Y. Yardım (yyardim@epoka.edu.al)
}

Received: 2 May 2013 - Published in Nat. Hazards Earth Syst. Sci. Discuss.: 31 October 2013

Revised: 17 December 2014 - Accepted: 4 May 2015 - Published: 26 May 2015

\begin{abstract}
Protecting the historical character of a valued structure during the assessment and damage repair process is a very challenging task for many engineers. Heritage protection is complicated by a lack of design details and restrictions on sample extraction needed to obtain accurate material properties and limited studies on the restoration of certain types of historical structures. This study aims to assess the effects of soil settlement on a structure's stress concentrations and the value of laser scanning techniques on structure analysis in obtaining correct data of settlement vs. deformation. Terrestrial laser scanner (TLS) data are used to analyse the 500-year-old historical structure of Naziresha's Mosque. The obtained TLS data allow an accurate definition of the imperfect geometry patterns lying on every side of the structure. The soil profile and general crack formation together with TLS measurement proves that the structure deformed toward the south façade, where a railway and motorway are also located. Stress concentration and mode period results have a considerable difference, which highlights earthquake vulnerability and failure mechanisms and changes the strategy of possible retrofitting.
\end{abstract}

\section{Introduction}

Finding a way to protect the historical character of a valued structure during the assessment and damage repairs process is a very challenging task for many engineers. Decay of materials, degradation from environmental conditions, seismic activities and geological phenomena, such as internal and external erosion and earthquakes, are some of the common external problems that affect historical structures and make the protection of cultural heritage a complex process (Riveiro et al., 2011). Modifications, wrong strengthening and restoration are man-made interventions to be considered when assessing vulnerabilities in historical structures. In addition, the restrictions on extracting samples to obtain accurate material properties and a limited number of studies on these types of structures increase the complexity of assessment (Pesci et al., 2012).

However, the accurate measurement of the current geometrical shape of a structure provides insights into structural vulnerabilities and degradation, such as the changes in the layout, existing crack propagations and settlements. Therefore, accurate geometrical measurements of historical structures with its imperfections have to be considered to model the present state and the effects of many interventions (Pesci et al., 2012).

The traditional methods of making measurements by hand are usually not sufficiently accurate (1), are time-consuming and labor-intensive (2) (Lahoz et al., 2006). The details of geometric data of historical structures including all the existing distress and geometric imperfections have been obtained by remote-sensing techniques (Lerones et al., 2010; Pesci et al., 2011, 2012) such as terrestrial laser scanning (TLS). The terrestrial techniques, applicable to 3-D modeling of infrastructures, consist of traditional topography, close-range photogrammetry and the TLS (Lubowiecka et al., 2009). This technique is able to provide a detailed 3-D model of the observed object, whose accuracy can reach from $5 \mathrm{~mm}$ to $100 \mathrm{~m}$ (Lahoz et al., 2006). The TSL 3-D model and digital ortho-image can easily be created using a generated 3-D point cloud and recorded digital images.

This study aims to demonstrate the use of precise geometric data obtained by methods in digital photogrammetry and terrestrial laser scanning on finite element model (FEM) 
structural analysis of a historical monument under seismic load. The structure was modeled with two different geometric data: as-built and assumed perfect geometry, which are obtained from precise geometric documentation with TLS and traditional geometric measurements. Then a structural analysis was carried out with the same material properties and type of seismic loads to compare the effects of two different geometric data on the structural assessment result. The effect of imperfect geometry, which is mainly caused by soil settlement due to different reasons, is obtained in terms of stress.

\section{Background}

The usage of the TLS technology to assess historical structures has increased in the last decade (Yastikli, 2007; González et al., 2010). Traditional measurement techniques often produce less reliable models because of the low accuracy of equipment, dependence on the technical ability of the operator and difficulties of architecture details. The accuracy concern became more important when the obtained geometric data were used as the base for FEM modeling of historical masonry structures (Lourenço et al., 2001; González et al., 2010; Ustundag et al. 2010).

There are many automated detection and visualization techniques used for geometrical data collection of historical structures. They can be classified on the basis of the sensor used: active sensor (i.e., laser scanning and 3-D range camera) and passive sensor (i.e., photogrammetry and videogrammetry) (Zhu and Brilakis, 2009; Fathi and Brilakis, 2011).

Recent studies show that the most suitable techniques for cultural heritage documentation and also 3-D modeling and visualization are photogrammetry and terrestrial laser scanning (Yastikli, 2007; Guarnieri et al., 2010; Prokop and Panholzer, 2009; Yerli et al., 2009; Bhatla et al., 2012). It is very difficult, or in some cases, quite impossible to obtain perfectly accurate geometric data with every single detail of a historical structure. Even though this is possible, many assumptions need to be taken during structural modeling, due to limitations of the software packages used and for the sake of simplification. However, this source of error may partially be avoided with experience and a deep knowledge in mechanics of materials and finite element modeling. In most of the cases, the geometric data documentation and assumptions were done by technical people having very little or no idea about structural modeling. Therefore, the impact of early assumptions on geometrical data and structural engineering simplifications during modeling could cause differences in FEM results. This difference could be more significant for a historical masonry structure because of the composition and the diversity of materials used (Lubowiecka et al., 2011; Pesci et al., 2012; Teza and Pesci, 2013). Such errors in the assessment may end up with ineffective or wrong strength- ening efforts. Eventually, the architectural authenticity of the structure could be disturbed.

The photogrammetry and terrestrial laser scanning techniques have also been used to carry out structural assessment; the models obtained by these techniques were converted into computational meshes to be able to generate FEM meshes (Pesci et al., 2012; Gordon and Lichti, 2005; Tang et al., 2010). The same techniques were applied on historical masonry arches by Riveiro et al. (2011) to obtain a precise geometric model for numerical analysis. The failure load in the numerical model with accurate geometry has proved to be higher by $12 \%$.

There is an increasing interest in using these techniques in several fields: heritage (Teza and Pesci, 2013; Hinzen et al., 2013; Tapete et al., 2013), medicine, geological survey and computer vision (Lubowiecka et al., 2011). Applications of the techniques have been used in construction safety (Prokop and Panholzer, 2009), load monitoring of structures (Pesci et al., 2012), disaster management (Mikoš et al., 2005) and construction progress evaluation (Turkan et al., 2012).

\section{The studied site and architectural features}

For a small country like Albania, acquiring the geometrical data of a structure with a laser scanner is not an easy task and usually expensive. Therefore, a special structure was selected to realize the benefits of this technology. Naziresha's Mosque is the only well-preserved historical structure in Elbasan, Albania (Fig. 1). It was built in the 1590s and is situated on a fenced plot in the southern suburb of Elbasan, a few hundred meters to the north of the Shkumbini River bank. The structure has a square $10.70 \mathrm{~m} \times 10.70 \mathrm{~m}$ plan and one cubicshaped central hall of $8.70 \mathrm{~m}$. The main load bearing system is comprised of $1 \mathrm{~m}$ thick stone walls and the main dome. It was built according to the late-Classical Ottoman style. Transition from the cube to the dome is provided by pendentives covered from outside by triangular shoulders. The dome rises over pendentives $14 \mathrm{~m}$ a.g.l. (above ground level). It is made of brick masonry $0.35 \mathrm{~m}$ thick and is covered with roof tiles (Fig. 2).

Masonry walls are made of rectangular cloisonné; neatly cut rectangular blocks formed with two layers of horizontally and two layers of vertically placed bricks. The windows are found on three floors. The ones in the first level are adorned with rectangular frames and crowned with a pointed arch with an adorned border. The windows of the other levels have the same characteristics, but are smaller in size and fewer in number. The minaret was damaged after an earthquake in 1920 and the upper section is missing (Mustafaraj, 2012).

The structure sits on alluvial deposits of the first terrace of the Shkumbini River (Fig. 1). The area is flat with an inclination of $1-2^{\circ}$ and the deposits consist of silts and clay intercalated by sand layers (upper part) and gravels in the lower 


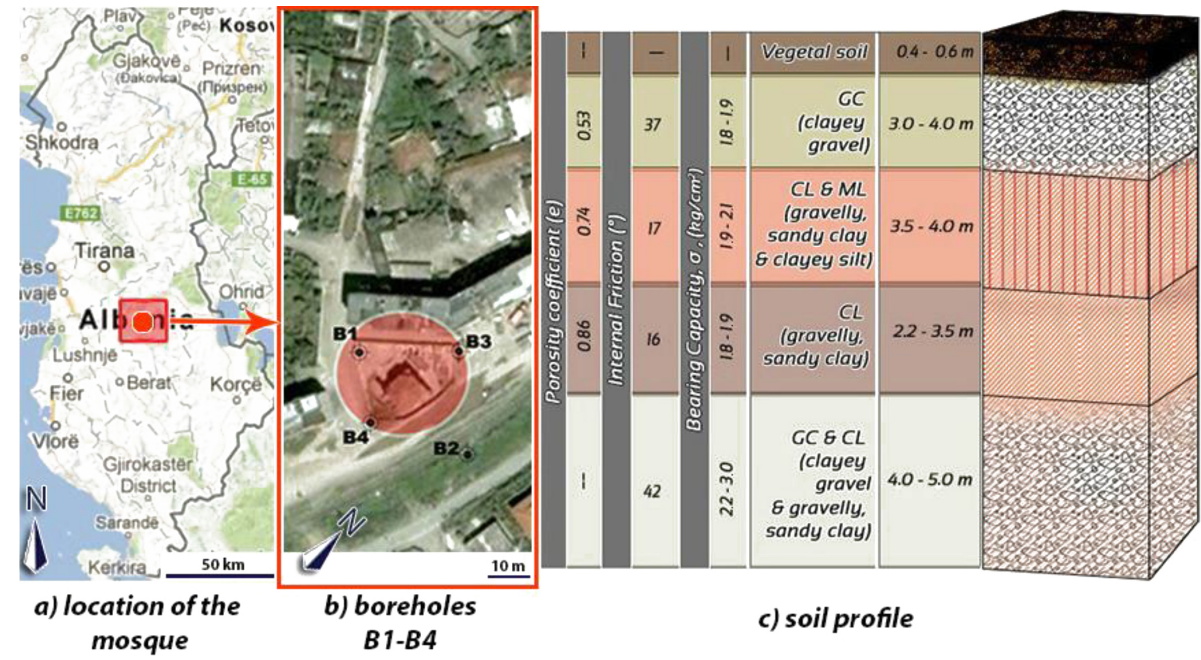

Figure 1. Location of the mosque (a), boreholes B1-B4 (b) and soil profile under the mosque (c).

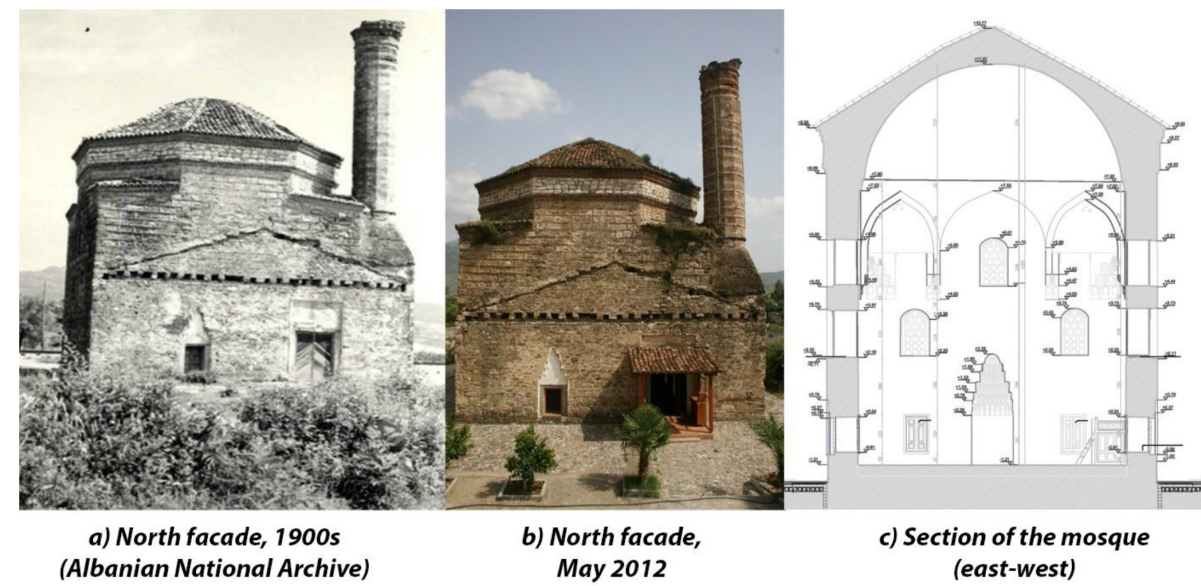

Figure 2. Main façade (a, b) and the cross section detail of the mosque (c).

part of the lithological profile. Field tests of boreholes, pits and ditch works were performed around the building. The boreholes depth varies from 10 to $20 \mathrm{~m}$ (see boreholes B1B4 in Fig. 1). Several tests were conducted to obtain general properties of soil under the structure. The laboratory works include grain size analysis, natural water content, bulk density, strength parameters, internal friction angle and cohesion based on ASTM. The underground water table is located $18.5-19.0 \mathrm{~m}$ below the surface level. In total, five geotechnical layers were seen from the field test (Table 1).

In addition to weak soil and high water level, terrain movements derived from the high seismicity affect the structure. Albania and the Balkan Peninsula are part of the AlpineMediterranean seismic belt. The released energy from the earthquakes of this belt is estimated to be about $15 \%$ of the total amount of the overall energy released from the earthquakes around the world (Papazachos, 1988). According to the statistical records of the seismic centres in Alba- nia and the neighboring countries, during 1900-2005, Albania was hit by 234 earthquakes with a magnitude of greater than 5 on the Richter scale. The earthquake that hit Elbasan in 1920 with $M_{\mathrm{S}}=6.5$ caused the complete collapse of 173 houses, 14 deaths, 300 injuries and the partial collapse of 1790 houses. This was the biggest earthquake to hit the city in the last century. The minaret of the Naziresha's Mosque and the surrounding castles' walls were partially destroyed in this earthquake (Aliaj et al., 2010).

Other influences on the structural conditions created by human intervention are the roads and the railway next to the structure. The railway which lies $6 \mathrm{~m}$ away from the structure was built in 1960 to connect the cities of Durrës and Korça. It is located $1 \mathrm{~m}$ a.g.l., and it is used 4 times a day with heavily loaded trains. In addition, a motorway was put in place just next to the structure (as shown in Fig. 3). Studies show that the presence of a railway at such a close distance to an unreinforced masonry structure may cause significant damage. 
Table 1. Geological properties of soil under the structure.

\begin{tabular}{lllllll}
\hline \multirow{2}{*}{ Description } & \multicolumn{5}{c}{ Composition (\%) } & Depth (m) \\
\cline { 3 - 6 } & & gravel & sand & silt & clay & \\
\hline Layer 1 & vegetal soil & - & - & - & - & $0.4-0.6$ \\
\hline Layer 2 & $\begin{array}{l}\text { gravel-sand-clay } \\
\text { mixture }\end{array}$ & $4.5-14.9$ & $23.7-33.7$ & $21.2-33.8$ & $4.5-14.9$ & $3.0-4.0$ \\
\hline Layer 3 & $\begin{array}{l}\text { inorganic silt, clay of } \\
\text { low-medium plasticity }\end{array}$ & - & $21.5-33.5$ & $51-65.2$ & $7.7-16.9$ & $3.5-4.0$ \\
\hline Layer 4 & $\begin{array}{l}\text { sand-gravel low- } \\
\text { medium plasticity }\end{array}$ & $1.1-9.1$ & $21.2-35$ & $51.2-61.4$ & 10.5 & $2.2-3.5$ \\
\hline Layer 5 & gravel-sand-silt mixture & $6.2-14.8$ & $18.9-29.7$ & $6.5-17.9$ & $2.4-3.8$ & $4.0-5.0$ \\
\hline
\end{tabular}

Koçak and Köksal (2010), in their study of Little Hagia Sophia Mosque, among other reasons, counted the effect of the railway as one of the big contributors to the settlement of the structure. The railway, which was operational for 50 years and lay $5 \mathrm{~m}$ away from the mosque, caused bricks to fall from the nearby wall when trains were passing by. The present settlement was measured towards the railway site of the structure. The significance of the railway's effect may increase with weak soil and a high water level (Koçak and Köksal, 2010).

\section{Methodology}

Data collection was enabled by a calibrated high-resolution digital camera (Nikon D90) firmly mounted onto the laser scanner (Optech ILRIS 3-D Intelligent Laser Ranging and Imaging System) together with Topcon GPT-3007 Total Station, which provided a combination of scan data and image data. The laser scanner facilitates the measurement procedure. It enables a field view of $360^{\circ}$ along horizontal and a $60^{\circ}$ view in vertical plane. In this way, a full panoramic view could be generated. The generated point cloud provided accurate details of the surface pattern of the structure and mapping coordinate system of the volume the structure covered. The Optech ILRIS 3-D long-range laser scanner has a beam divergence (in mrad), $D(r)=1.7 \times 10^{-4} \times r+0.012$, where $r$ is the acquisition distance in meters.

Depending on the details sought and the surrounding obstructions, the scanning process was carried out at a horizontal distance of 10-20 m from the structure. A total of 15 scans were taken from five external and two internal stations as shown in Fig. 4. The stop spacing was set to $1 \mathrm{~cm}$ at $50 \mathrm{~m}$ to obtain better cloud points details and the total process took approximately $8 \mathrm{~h}$.

The structure surface was scanned using the laser scanner with high resolution. A laser beam is used to obtain the geometric coordinates $(x, y, z)$ of points at regular intervals on the visible surface of the structure. Then a point cloud

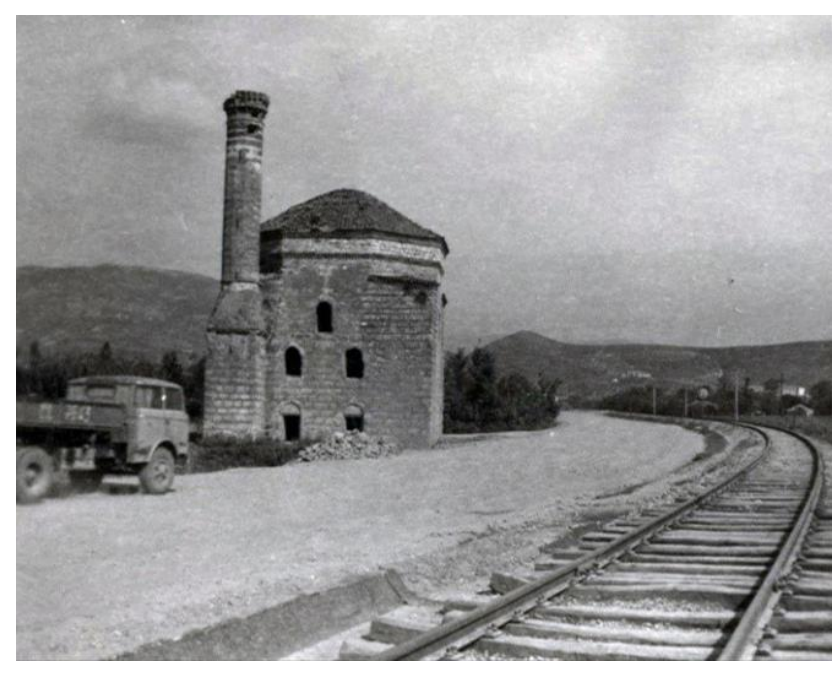

Figure 3. Motorway and rail way built next to structure 1950s, (obtained from Albanian National Archive).

is obtained based on the adjustment and sensitivity of used equipment. A color imaging device such as a 3-D camera is used together with the scanner to project the structure's geometric data onto the image obtained for each scanned point. The obtained resulting point cloud is later purified in order to obtain the best representative surface of a polygonal model consisting of a triangle mesh with PolyWorks (InnovMetric Inc., 2012) software. The purifying process is conducted by setting a measuring threshold collecting bad data which usually occur during the scanning process due to the time flight of light rays.

The second stage of the geometric data representation with TSL is carried out in the office. Obtained data were merged using the PolyWorks commercial software. The software allows the input data to be accurately purified. The produced triangular meshes control model details. Complete model sizes were significantly reduced (up to $40 \%$ ) while protecting surface details. With the help of this software, the model 


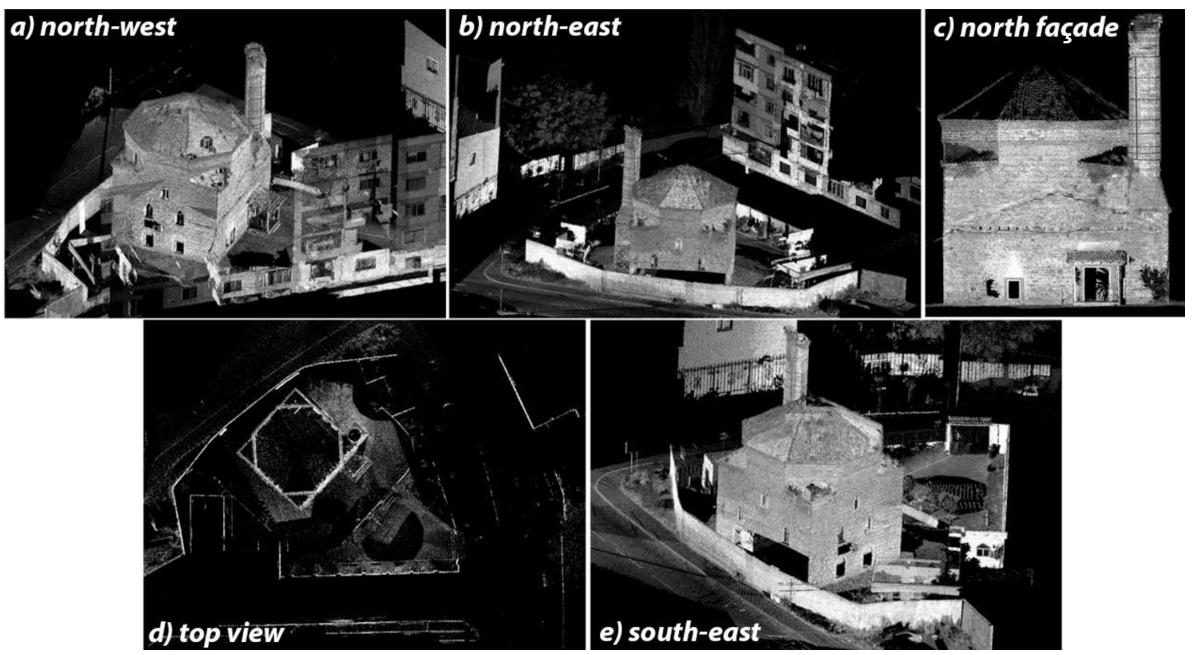

Figure 4. Laser scanner view of the structure from different scan stations (May 2012): (a) northwest, (b) northeast, (c) north façade, (d) top view, (e) southeast corner.

can be detailed where needed. Finally, three-dimensional digital models were obtained.

An FEM modeling was carried out to demonstrate the behavior of the structure based on the rough shape and a more accurate shape. The analysis was carried out using SAP2000 v.15.0 software (CSI, 2011), based on Eurocode 8 (EN1998-1, 2004), with consideration of the local earthquake code (KTP-N2, 1989). The elements and material were chosen to obtain the most realistic simulation of the structure's behavior. In order to validate the accuracy of the assumed results, the modal frequencies were compared with previous studies. The most difficult part in modeling a historical monument is obtaining the right material characteristics. Since Naziresha's mosque is a very old structure, the limitation of knowledge is unavoidable due to the lack of information or documentation of types of construction materials, original architectural plans, maintenance schedule, modification and restoration phases during its lifetime, etc. Additionally, obtaining the required number of samples for testing is not possible as it is a cultural monument of the first category protected by law. Uncertainty may come from equipment accuracy and measurement errors which may be overcome by using tools with higher accuracy. In this study, the obstacles related to acquisition of an accurate geometry of the structure are overpassed by making use of TLS and photogrammetry, having a geometrical model as close as reality. The model generated from TLS showed that there was a variation in the thickness of the load bearing walls from 0.8 to $1.1 \mathrm{~m}$, whereas in the architectural plan they are described as $1 \mathrm{~m}$ thick.

In the model, the modulus of elasticity and characteristic stresses were defined according to previous studies in which experimental tests were carried out to evaluate the charac-
Table 2. Assumed material properties of the FE model.

\begin{tabular}{lll}
\hline & Brick & Stone \\
\hline Unit weight, $\gamma\left(\mathrm{kN} \mathrm{m}^{-3}\right)$ & 17 & 21 \\
Modulus of elasticity, $E(\mathrm{MPa})$ & 2100 & 1740 \\
Void ratio, $v$ & 0.2 & 0.2 \\
Tensile strength $(\mathrm{MPa})$ & 0.564 & 1.42 \\
Compressive strength $(\mathrm{MPa})$ & 1.03 & 4.06
\end{tabular}

teristic properties of the materials (Faella et al., 2004). The material properties used for the model are shown in Table 2.

The masonry walls and the dome were modeled using macro-modeling (masonry units and mortar layers are considered a continuum, where masonry is isotropic, homogeneous and shows elastic behavior) with shell elements.

A response spectrum analysis was performed to obtain critical stresses. This analysis is a statistical method used to determine the likely maximum response of the structure under a specific seismic loading. It is defined as a set of values (acceleration, velocity or displacement) consisting of the maximum responses of a single mass oscillator that varies with natural frequencies and a given damping. In this analysis, a spectrum of pseudo-spectral acceleration versus period is considered. This response indicates a statistical magnitude of the likely maximum response for the considered earthquake (CSI, 2011).

In order to study the effects of the ground motion excitations on the structure, it is necessary to measure the intensity of the motion. Hence, a response spectrum analysis was performed based on EN1998-1 with a considering local code of KTP-89. Peak ground acceleration based on obtained soil data was chosen as $0.25 \mathrm{~g}$. 


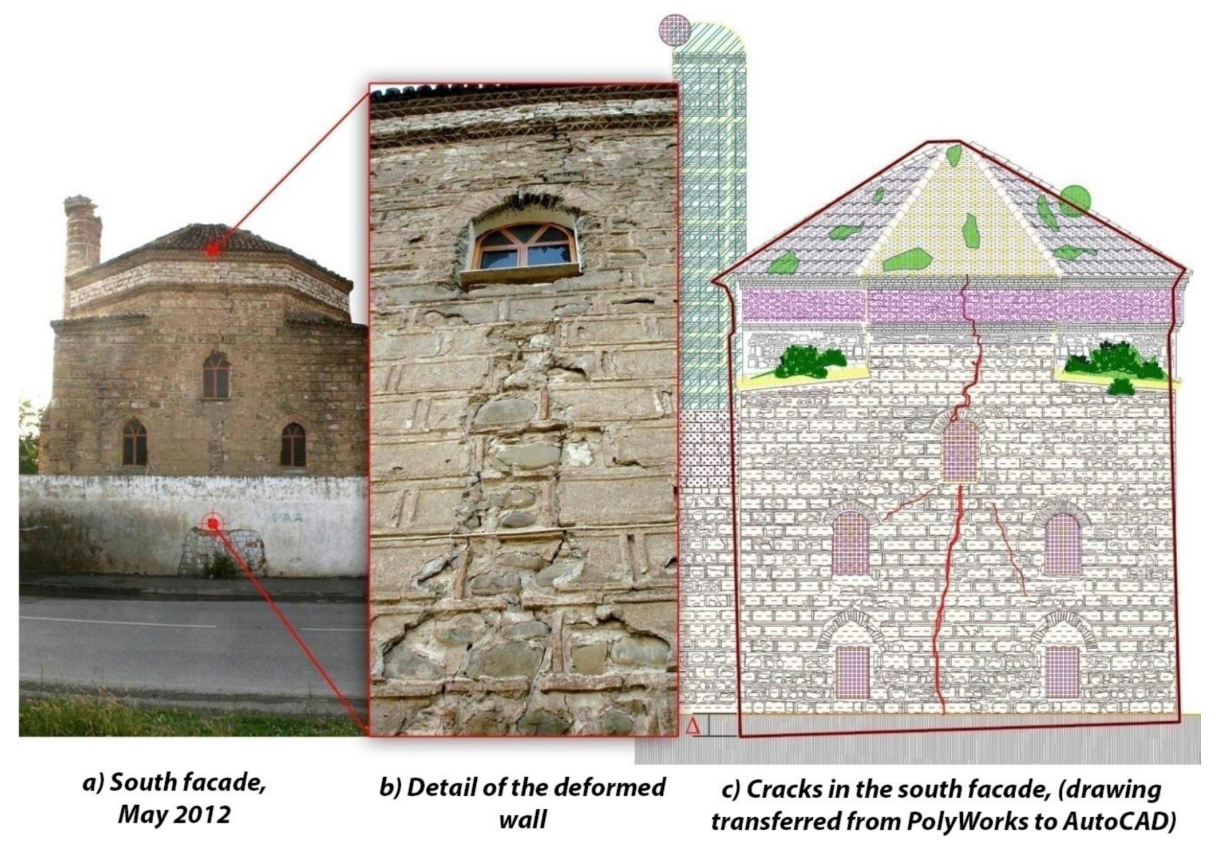

Figure 5. Cracks due to differential settlement $(\Delta=18 \mathrm{~cm})$ in the south façade (May 2012).

\section{Results and discussion}

Representation of geometric imperfections is not uniform along the structure; therefore, it is not easy to describe all effects of such deformations. The recorded deformation has been progressing for centuries. The morphological features of the site and soil profile have been playing a key role for this imperfection in geometry. Seismic activities and constant vibration because of external impact accelerate and form this deformation. Final shapes are formed with a combination of these events with material characteristics. However, the soil profile and general crack formation together with the TSL measurement proves that the structure is deformed toward the south façade of the mosque where the railway and motorway are also located.

The main reasons for this complexity rise from a variety of internal and external influences. Based on the field study, the measured geometric imperfection of the structure is primarily caused by differential settlement of the foundation due to the soil profile. The soil layers are very suitable to produce internal erosion with its different void ratio and particle size along the vertical. This type of settlement is triggered by earthquakes, frequent changes in underground water level caused by the river base change, changes of ground water level due to surrounding drainage system and constant vibrations created by the adjacent motorway and railway. In addition to the above-mentioned factors, the maintenance that had been carried out at different times during the lifespan of the mosque with different materials whose characteristics do not match the original materials could be a secondary or an additional cause of imperfect geometry.
The laser scanner data were transferred to AutoCAD (Autodesk Inc., 2012) and Sap2000 (CSI, 2011) software to figure out a detailed representation of the geometric model. Several optimizations and approximations were carried out during this transformation between the software programs. The main aim of this optimization is to avoid several types of misrepresentations and error such as noise due to dust, vegetation and cracks. Based on the detailed scan, the southwestern corner of the structure has settled down to $18 \mathrm{~cm}$ (Figs. 5 and 6). This corner is directly facing the motorway and railway that passes next to the structure.

With the help of a laser scanner acquisition, the obtained geometric imperfection results in an increase in stress concentrations under seismic load. This imperfection changes the possible failure mechanisms and threatens the historical monument if an earthquake were to occur in the area. With respect to the corresponding models with more accurate geometry, the maximum tensile stress that occurs in the structure is $0.361 \mathrm{MPa}$ under static load, $0.801 \mathrm{MPa}$ under seismic load and $0.144 \mathrm{~s}$ for the first mode. On the other hand, the actual geometric model gives $0.774 \mathrm{MPa}$ under static load, 1.4 MPa under seismic load and $0.174 \mathrm{~s}$ for the first mode. The existing structural defects associated with the stress results are shown in Fig. 7; as expected, stress concentrations were recorded at the corners of openings and wall connection locations.

The cracks on the existing structure and stress concentration in the model verify this method. The key findings from a comparison of stress concentrations due to response spectrum analysis are given in Fig. 7. The imperfect geometry due to settlement directly affects stress concentration values 


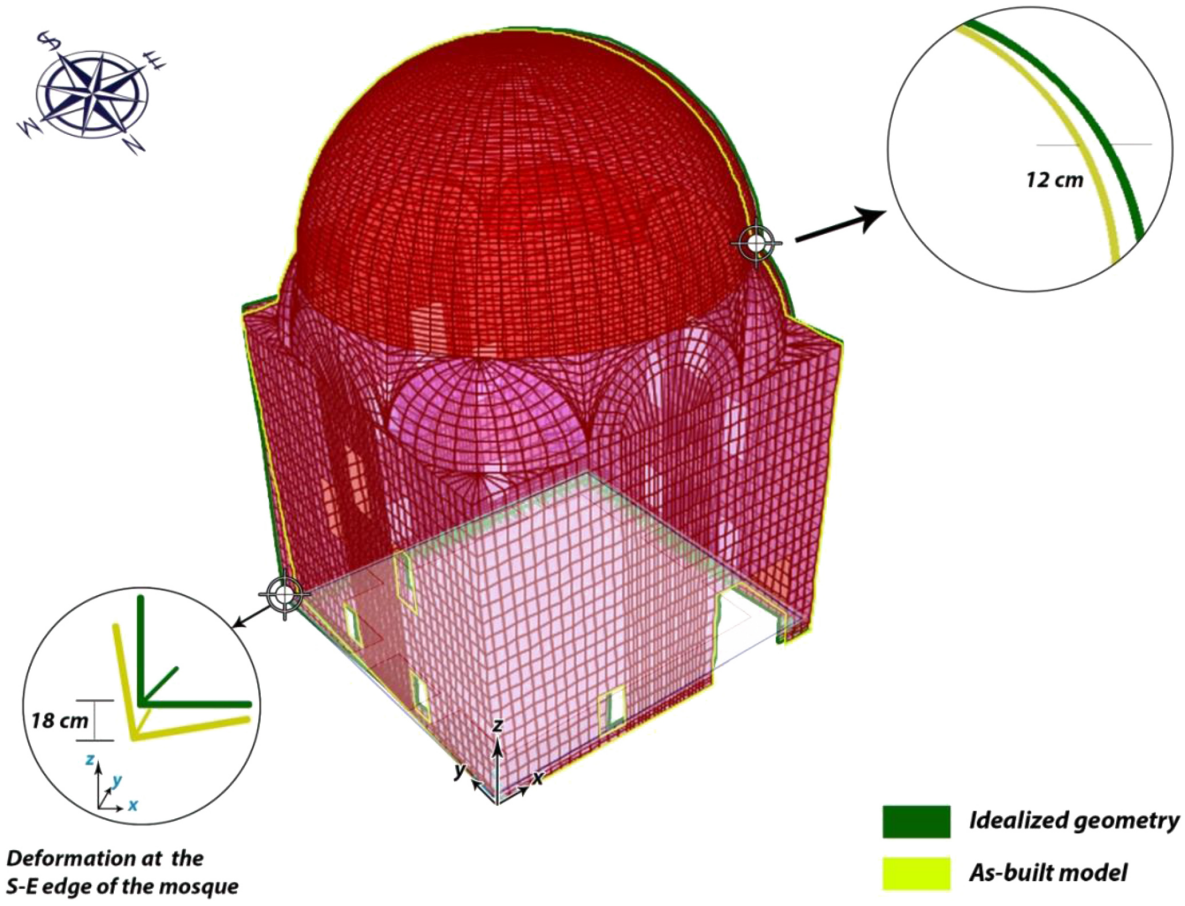

Figure 6. Deformation of the structure at the southeastern corner and east of the dome.

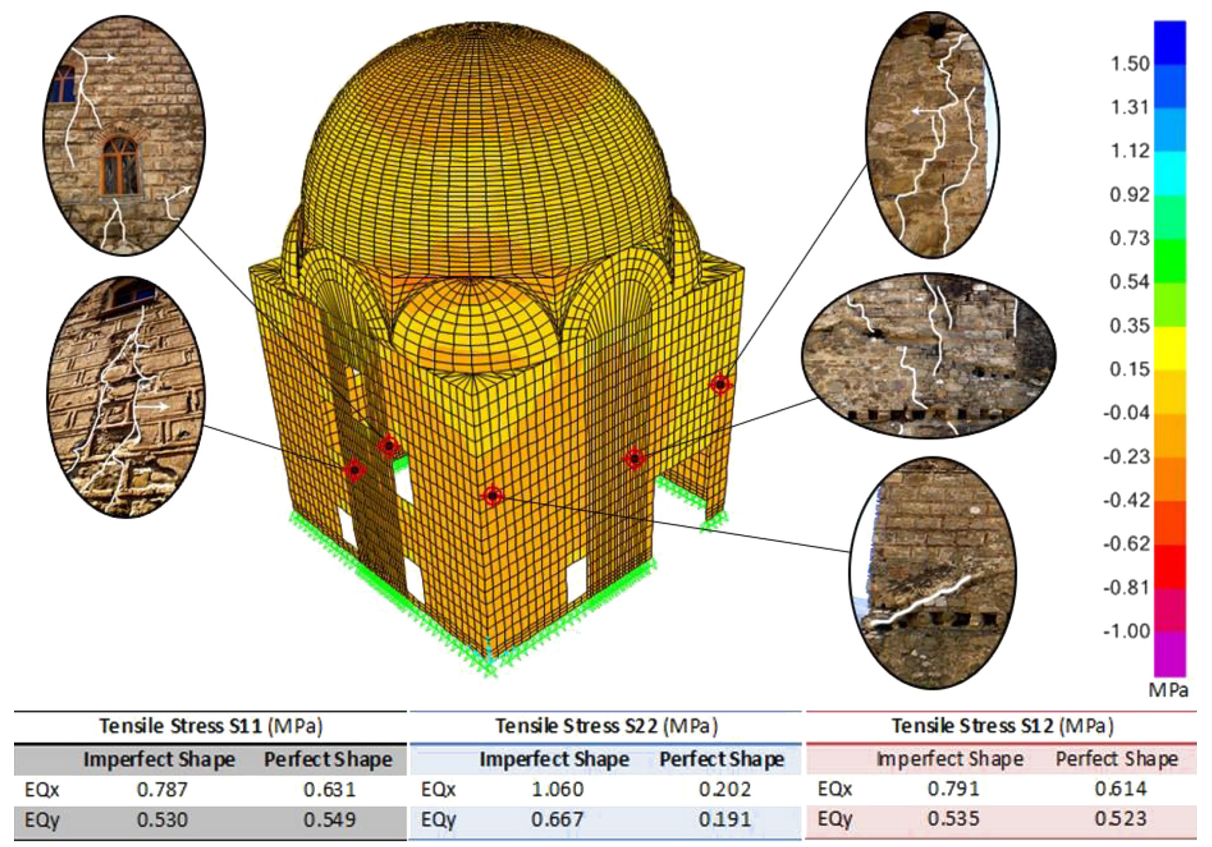

Figure 7. Stress difference for accurate and imperfect geometric model under combined dead and earthquake loading (stress values obtained from SAP2000).

which are the key values to drawing retrofitting strategies for the historical monument. The comparison between the modes shows that the imperfect geometry has considerable influence on modes as well. The periods for every mode are summarized in Fig. 8. Stress concentration and the mode pe- riod difference between the rough and accurate models give good evidence of the extreme importance of the definition of the correct geometry for this type of structure. 




Figure 8. First five modes for as-built and assumed perfect geometry (SAP2000).

\section{Conclusions}

There were two main topics of interest in this study: (1) the impact of the soil settlement on a structure's stress concentrations and (2) the influence of the laser scanning techniques on structure modeling by obtaining the correct data of settlement vs. deformation. The method used to analyse the structure based on TLS data allows an accurate definition of the imperfect geometry patterns on every side of the structure. The comparison of two models presented significant stress values along the structure, hence, a different crack pattern. Additionally, the morphological and geological information seem to support the TLS-based model showing a deformation resulting from soil settlement. This settlement has been a consequence of cumulative effects of soil profiles and also seismic events which have occurred from the 1600s to today and man-made vibrations due to vehicular and train traffic. Final shapes are formed by combining these events with material characteristics. Therefore, TLS-measured imperfections in geometry are not in a uniform shape to be attributed to one activity. However, soil profile and general crack formation together with TSL measurement suggests that the structure deformed toward the south façade, where a railway and mo- torway are also located. The stress concentration and mode period results have a considerable difference which highlights the failure mechanism and the mosque's vulnerability to earthquakes in the area and suggests the correct strategy for possible retrofitting. The proposed method to detect geometric imperfection by TLS and incorporating the obtained data into a structure model can be taken into account for future restoration, retrofitting and acting on localized anomalies such as cracks and settlement.

Acknowledgements. The authors wish to thank AST-Ahmet Soner Toğanaş Bureau of Architecture, Ankara, for providing us with useful architectural data of the mosque and their help with laser scanning process.

Edited by: B. D. Malamud

Reviewed by: four anonymous referees

\section{References}

Aliaj, S., Muço, B., and Sulstarova, E.: Sizmiciteti, sizmotektonika dhe vleresimi i rrezikut sizmik ne Shqipëri, (Albanian), Academy of Sciences of Albania, Tirana, Albania, 2010. 
Autodesk Inc.: http://www.autodesk.com/, last access: 28 November 2012.

Bhatla, A., Choe, S. Y., Fierro, O., and Leite, F.: Evaluation of accuracy of as-built 3D modeling from photos taken by handheld digital cameras, Automat. Construct., 28, 116-127, 2012.

CSI, SAP2000 v-15.0: Integrated finite element analysis and design of structures basic analysis reference manual, Computers and Structures Inc, Berkeley, California, USA, 2011.

EN 1998-1: European seismic design code, Design of structures for earthquake resistance, Part 1: General rules seismic actions and rules for buildings, 2004.

Faella, C., Martinelli, E., Nigro, E., and Paciello, S.: Tuff masonry walls strengthened with a new kind of C-FRP sheet: Experimental tests and analysis, Paper No. 923, 13th WCEE, 1-6 August 2004, Vancouver, 2004.

Fathi, H. and Brilakis, I.: Automated Sparse 3D Point Cloud Generation of Infrastructure using its Distinctive Visual Features, Adv. Eng. Inform., 25, 760-770, 2011.

González, J. A., Rodríguez, B. R., Aguilera, D. G., and Brea, R. T.: Terrestrial laser scanning intensity data applied to damage detection for historical buildings, J. Arch. Sci., 37, 3037-3047, 2010.

Gordon, S. J. and Lichti, D. D.: Modeling terrestrial laser scanner data for precise structural deformation measurement, J. Surv. Eng., 133, 72-80, 2005.

Guarnieri, A., Pirotti, F., and Vettore, A.: Cultural heritage interactive 3-D models on the web: an approach using open source and free software, J. Cult. Herit., 11, 350-353, 2010.

Hinzen, K. G., Schreiber, S., Fleischer, C., Reamer, S. K., and Isabel Wiosna, I.: Archeoseismic study of damage in Roman and Medieval structures in the center of Cologne, Germany, J. Seismol., 17, 399-424, 2013.

InnovMetric Inc: http://www.innovmetric.com/polyworks/ 3D-scanners/home.aspx?lang=en, last access: 12 December 2012.

Koçak, A. and Köksal, K.: An example for determining the cause of damage in historical buildings: Little Hagia Sophia (Church of St. Sergius and Bacchus) - Istanbul, Turkey, Eng. Fail. Anal., 17, 926-937, 2010.

KTP-N2: Earthquake Resistant Design Regulations, Academy of Science of Albania, Tirana, Albania 1989.

Lahoz, J. G., Aguilera, D. G., Finat, J., Martínez, J., Fernández, J., and José, S. J.: Terrestrial laser scanning metric control: assessment of metric accuracy for cultural heritage modeling, 10 Proc. ISPRS Commission V Symp. Dresden, Germany, 2006.

Lerones, P. M., Fernández, J. L., Gil, A. M., Bermejo, J. G. G. B., and Casanova, E. Z.: A practical approach to making accurate 3-D layouts of interesting cultural heritage sites through digital models, J. Cult. Herit., 11, 1-9, 2010.

Lourenço, P. B., Mele, E., Gatto, D., and Luca, A. D.: Structural analysis of basilica churches: a case study, Historical Constructions, Guimarães, Portugal, 729-738, 2001.

Lubowiecka, I., Armesto, J., Arias, P., and Lorenzo, H.: Historic bridge modelling using laser scanning, ground penetrating radar and finite element methods in the context of structural dynamics, J. Eng. Str., 31, 2667-2676, 2009.

Lubowiecka, I., Arias, P., Riveiro, B., and Solla, M.: Multidisciplinary approach to the assessment of historic structures based on the case of a masonry bridge in Galicia (Spain), Comput. Struct., 89, 1615-1627, 2011.
Mikoš, M., Vidmar, A., and Brilly, M.: Using a laser measurement system for monitoring morphological changes on the Strug rock fall, Slovenia, Nat. Hazards Earth Syst. Sci., 5, 143-153, doi:10.5194/nhess-5-143-2005, 2005.

Mustafaraj, E.: A case study on structural assessment of Ottoman mosques in Albania, MSc Thesis, Epoka University, Tirana, Albania, 2012.

Papazachos, B. C: Active tectonics in Aegean and surrounding area, Proceedings Summer School on Seismic Hazard in Mediterranean Regions, 21-30 July 1986, Strasbourg, France, 301-331, 1988.

Pesci, A., Casula, G., and Boschi, E.: Laser scanning the Garisenda and Asinelli towers in Bologna (Italy): detailed deformation patterns of two ancient leaning buildings, J. Cult. Herit., 12, 117127, 2011.

Pesci, A., Bonali, E., Galli, C., and Boschi, E.: Laser scanning and digital imaging for the investigation of an ancient building: Palazzo d'Accursio study case (Bologna, Italy), J. Cult. Herit., 13, 215-220, 2012.

Prokop, A. and Panholzer, H.: Assessing the capability of terrestrial laser scanning for monitoring slow moving landslides, Nat. Hazards Earth Syst. Sci., 9, 1921-1928, doi:10.5194/nhess-9-19212009, 2009.

Riveiro, B., Caamaño, J. C., Arias, P., and Sanz, E.: Photogrammetric 3-D modelling and mechanical analysis of masonry arches: an approach based on a discontinuous model of voussoirs, Autom. Constr., 20, 380-388, 2011.

Tang, P., Huber, D., Akinci, B., Lipman, R., and Lytle, A.: Automatic reconstruction of as-built building information models from laser-scanned point clouds: a review of related techniques, Autom. Constr., 19, 829-843, 2010.

Tapete, D., Casagli, N., Luzi, G., Fanti, R., Gigli, G., and Leva, D.: Integrating radar and laser-based remote sensing techniques for monitoring structural deformation of archaeological heritage, J. Arch. Sci., 40, 176-189, doi:10.1016/j.jas.2012.07.024, 2013.

Teza, G. and Pesci, A.: Geometric characterization of a cylindershaped structure from laser scanner data: development of an analysis tool and its use on a leaning bell tower, J. Cult. Herit., 14, 411-423, 2013.

Turkan, Y., Bosche, F., Haas, C. T., and Haas, R.: Automated progress tracking using 4-D schedule and 3-D sensing technologies, Autom. Constr., 22, 414-421, 2012.

Ustundag, C., Sesigur, H., and Cili, F.: Seismic evaluation and retrofit of the 16th century Mihrimah Sultan Mosque, 14th European Conf. on Earthquake Eng., 30 August-9 September 2010, Ohrid, F. Y. R. Macedonia, 2010.

Yastikli, N.: Documentation of cultural heritage using digital photogrammetry and laser scanning, J. Cult. Herit., 8, 423-427, 2007.

Yerli, B., Schreiber, S., Veen, J. H. T., and Sintubin, M.: Testing the hypothesis of earthquake related damage in structures in the Lycian ancient city of Pinara, SW Turkey, 1st INQUAIGCP-567 Int. Workshop on Earthq. Archaeo. Palaeoseism., 7-13 September 2009, Baleo Claudia, Spain, 173-176, 2009.

Zhu, A. and Brilakis, I.: Comparison of optical sensor-based spatial data collection techniques for civil infrastructure modeling, J. Comput. Civ. Eng., 23, 170-178, 2009. 